\title{
LA GASTRONOMÍA COLOMBIANA. ATRACTIVO TURÍSTICO EN CRECIMIENTO
}

\section{THE COLOMBIAN GASTRONOMY. A GROWING TOURIST ATTRACTION}

\author{
Rodrigo Naranjo Arango*, Gloria Naranjo Africano** \\ Ader Ricardo Leones Viana***
}

\footnotetext{
* Doctor en Administración de Negocios. Director Grupo de Investigaciones Estrategia \& Liderazgo. Barranquilla, Colombia.

** Candidata a Doctor en Administración. Universidad del Norte. Email: gnaranjo45@gmail.com

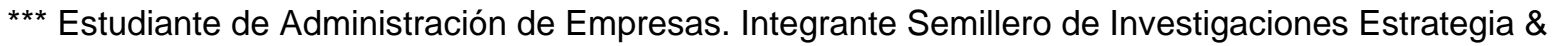
Liderazgo. Email: aderleonesv@hotmail.com
}

Dirección para recibir correspondencia: rnaranjo@naranjoconsulting.com 


\section{RESUMEN}

OBJETIVO: Demostrar que la gastronomía colombiana se ha convertido en un atractivo para el turismo, con un potencial de crecimiento y desarrollo.

MATERIAL Y MÉTODO: Tipo descriptivo y se apoyó en revisión de un material bastante amplio como fuentes secundarias, que permitieran construir una caracterización con base en la literatura de como la gastronomía constituye un atractivo turístico en la actualidad.

RESULTADOS: Se pudieron evidenciar datos significativos con relación a los platos más representativos de cada región, del mismo modo se encontraron hallazgos como cifras muy dicientes acerca de cómo ha avanzado el sector gastronómico dentro del país y las potencialidades que éste tiene.

CONCLUSIONES: La gastronomía del país es uno de los grandes pilares de la economía pues las cifras y los resultados obtenidos hablan por sí solos, y que de ser potencializado se estarían generando resultados extraordinarios no solo a nivel de crecimiento sino de desarrollo económico.

PALABRAS CLAVE: Gastronomía. Turismo. Desarrollo de marca.

\section{ABSTRACT}

OBJECTIVE: To demonstrate that the Colombian gastronomy has become an attraction for tourism with a growing and development potential.

MATERIAL AND METHOD: Descriptive type and it was supported on the review of abundant materials as secondary sources, that allowed to build a characterization based on the literature of how gastronomy represents a tourist attraction today.

RESULTS: It was possible to show significant data in relation to the most representative dishes of each region, in the same way, evidence such as explicit figures about the potentialities of the gastronomy and how the gastronomic sector has advanced within the country was found

CONCLUSIONS: The gastronomy of the country is one of the greatest foundations of the economy since the figures and the results obtained speak for themselves, and if the gastronomy 
LA GASTRONOMÍA COLOMBIANA. ATRACTIVO TURÍSTICO EN CRECIMIENTO

were to be potentialized, extraordinary results would be generated not only in terms of growth but also in terms of economic development.

Keywords: Gastronomy. Tourism. Brand development.

\section{INTRODUCCIÓN}

La gastronomía es considerada un arte, cada plato de comida elaborado es una fiel muestra de las costumbres, las tradiciones, la cultura de cada región; es así como la gastronomía se está convirtiendo en uno de los factores clave de atracción, tanto para definir la competitividad de destinos turisticos como para la promoción de los mismos (López-Guzmán \& Sánchez Cañizares, 2012).

Particularmente, la comida colombiana se caracteriza por se mestiza, así como el país presenta diferentes factores étnicos, no existe una sola gastronomía, sino diversas gastronomías regionales. Cada una de las regiones posee sus especialidades, en las cuales se mezclan ingrendientes indígenas, españoles, con formas de preparación africanas, árabes y españolas, que han sido un legado que se conserva hasta hoy (Bogotá turismo, 2016). La gastronomía de Colombia cuenta con gran reconocimiento no solo a nivel local, puesto que ya ha traspasado las fronteras y hoy en día es ampliamente reconocida internacionalmente, lo que la hace un referente en materia culinaria.

La diversidad étnica, geográfica y cultural, han llevado a Colombia a ser un país reconocido gastronómicamente hablando; esto asociado a un alto nivel de variedad, debido a que cuenta con seis regiones; cada región mantiene un alto nivel con respecto a la variedad gastronómica, considerando que presentan diferentes especialidades, donde los ingredientes usados para la elaboración de cada plato son distintos en muchos casos, lo que provoca una mezcla de sensaciones y sabores al momento de degustarlos (El tiempo, 2010). Esta amplia variedad de opciones, en ocasiones genera una gran amalgama de opciones que no permite establecer una identidad gastronómica en el país; y que se establece una visión fraccionada asociada a cada región que tiene su propio portafolio gastronómico.

El turista internacional considera que lo mejor de viajar es la amabilidad de la gente, la solidaridad, valoran los sitios turísticos y sobre todo la gastronomía nacional. Últimamente el turista está buscando tener una relación mas directa con las comunidades que visita, desea NARANJO-ARANGO R., NARANJO-AFRICANO G., LEONES-VIANA A. R. 


\section{LA GASTRONOMÍA COLOMBIANA. ATRACTIVO TURÍSTICO EN CRECIMIENTO}

vivir sus costumbres y compenetrarse aún mas con la cultura y las tradiciones, es por esto que la gastronomía se convierte en un factor determinante que puede contribuir no solo en la obtención de ganancias sino que también puede brindar estabilidad económica al país, así lo afirma (Dinero, 2013).

Teniendo en cuenta el auge que se está viviendo en torno al turismo gastronómico, se ha visto a la gastronomía como un producto turístico que debe ser explotado al máximo, debido a que Colombia tiene todo el potencial para convertise en uno de los destinos del turismo gastronómico mas importantes del mundo. Es por ello, que ésta investigación se enmarca dentro del hecho de reconocer la importancia que tiene la gastronomía como un atractivo turístico de Colombia y caracterizar los principales aspectos tanto económicos, sociales y culturales asociados al mismo.

\section{MATERIAL Y MÉTODO}

Este es un estudio descriptivo, soportado en el análisis de fuentes secundarias basado en artículos, libros, información disponible en la web, establecido en la siguiente estructura: (i) se tomó en consideración el aporte que hace cada de una de las regiones en materia del arte culinario, lo que sin duda alguna se ha convertido en uno de los principales elementos que cada vez mas está llamando la atención de los turistas para visitar a Colombia; (ii) se identificó la contribución que el sector grastronómico realiza al producto interno bruto y a la economia del pais.

Lo anterior, permitirá comprender que realmente la gastronomía se ha convertido en un factor, que sin dudas atrae a mas personas; con el paso del tiempo, Colombia ha ido consolidando su imagen en los mercados internacionales como uno de los países con mayor riqueza gastronómica en el mundo.

\section{RESULTADOS}

\section{El turismo gastronómico}

En cuanto a la gastronomía como atractivo turístico, es importante realizar las siguientes distinciones: (i) los turistas que se alimentan porque se encuentran fuera de su lugar de residencia habitual y (ii) los turistas cuya selección del destino se relaciona directamente con la gastronomía (Mitchell \& Hall, 2003). Con base a esto entonces se puede definir como turismo gastronómico la visita a productores primarios o secundarios de alimentos, participación en NARANJO-ARANGO R., NARANJO-AFRICANO G., LEONES-VIANA A. R. 
LA GASTRONOMÍA COLOMBIANA. ATRACTIVO TURÍSTICO EN CRECIMIENTO

festivales gastronómicos y búsqueda de restaurantes o lugares específicos, donde la degustación de alimentos y toda experiencia inherente es la razón principal para viajar (Hall \& Mitchell, 2002).

Uno de los recursos turísticos potenciados en las dos últimas décadas es el gastronómico, aproximadamente un $25 \%$ de los gastos de los turistas es en alimentación, al incluir los consumidos y los que compran para el regreso jugando un papel clave para el mantenimiento del empleo agrario, agroindustrial, de bares y restaurantes, comercios (Gaona, 1999).

En el turismo gastronómico, los productos alimenticios y las especialidades culinarias, se convierten en vehículo de acercamiento cultural en la medida en que se configuran como elementos identificadores de pueblos y territorios. Esta nueva forma de aproximación a la cultura de un lugar, región o país tiene un carácter más activo y vivencial, lúdico y festivo y, a priori, procura un desarrollo sostenible que pasa por: a) asegurar el mantenimiento de los recursos turísticos; b) mejorar los niveles de bienestar de la población que se encarga de su producción y comercialización y, por último, c) garantizar la satisfacción de la experiencia turística por la vía de la calidad (Feo Parrondo, 2005).

Particularmente, en el marco de una experiencia turística, el turista se encuentra con motivaciones principales (cuando visita un lugar con una motivación culinaria) y secundarias (aunque no tiene como prioridad lo gastronomico, lo disfruta en su viaje), las cuales se interelacionan por contraste (cuando disfruta de una gastronomia completamente diferente al de su lugar de origen), intensificacion (cuando refuerza los alimentos que ingiere en su lugar de residencia) y extensión (cuando se alimenta de lo que come en su lugar de origen) (Quan \& Wang, 2004) .

La relación de contraste, permite la incorporación de determinadas ciudades a circuitos internacionales, como ciudades claves de destino turísticos, debido a la fuerza y reconocimiento que tienen para los viajeros (López-Guzmán \& Sánchez Cañizares, 2012).

\section{Turismo gastronómico en Colombia y su aporte a la economía colombiana}

En los últimos años, Colombia se ha convertido en uno de los destinos turísticos más apetecidos por los extranjeros, no solo por ser un territorio rico en lugares para visitar y culturas por conocer, sino que además, su gastromonía se ha convertido en uno de los elementos mas significativos para atraer a las personas. Según la ministra de Comercio, Industria y Turismo, 


\section{LA GASTRONOMÍA COLOMBIANA. ATRACTIVO TURÍSTICO EN CRECIMIENTO}

para el año 2017, más de 6,5 millones de turistas visitaron a Colombia, y vale la pena destacar, que un gran porcentaje de ellos ingresó por los eventos gastronomicos, asi lo asevera (El espectador, 2018). Esta información coloca en evidencia que que el sector gastronómico tiene el potencial de ser uno de los ejes importantes en la economía colombiana.

En este sentido, se puede afirmar que la gastronomía colombiana se ha convertido en uno de los principales impulsores del turismo y en empleo; es por ello, que se considera que el arte culinario es una forma de mostrar al mundo nuestra cultura y valores (Mincomercio, 2012). Como evidencia de ello, se encontró que el sector gastronómico en Colombia ha venido haciendo aportes significativos a la economía nacional.

(Ruíz, 2013) afirma que en los últimos 10 años las ventas por consumo de comida por fuera del hogar han incrementado en 151,89\%; ésta cifra evidencia como ha ido creciendo el sector de los restaurantes; un crecimiento acelerado representado en ventas cercanas a los 24 billones en el año 2012, superando asímismo al año 2011 en la cual las ventas alcanzadas fueron de 20 billones. Este crecimiento, ha sido de tal forma que para inicios del año 2000, las ventas registradas eran de 7,9 billones, a lo que 10 años mas tarde, para el 2010 las ventas ascendieron a 19,9 billones.

Asociado a lo anterior, hace algunos años, el sector gastronómico se viene incluyendo dentro de los sectores que mas aportan al PIB del país, superando en algunas ocasiones al sector de la educación y las telecomunicaciones esto según (Ruíz, 2013). De igual forma, se tiene que en el año 2015 alcanzó un crecimiento mayor al 22\% y especialmente, en el sector turismo, la gastronomía aportó 3,6\%, cifra que ha venido creciendo progresivamente, según (Garzón, 2015).

Por otro lado, según el Dane, el sector hotelero y de restaurantes fue el segundo con mayor crecimiento en el año 2015 (El tiempo, 2016). Por lo que el sector no solo está haciendo aportes en términos de crecimiento económico, sino que también esta aportando a la generación de empelo, en el año 2015 el sector restaurantes creció con 90,000 restaurantes cada uno tiene como mínimo 10 empleados, lo que se traduce finalmente en oportunidad para que muchos salgan del desempleo y se logren vincular a la vida laboral, mejorando en este sentido los indicadores de desarrollo económico (Garzón, 2015).

Para el año 2016, las cifras han tenido un auge mayor, puesto que (Vanguardia.com, 2016) asevera que el sector gastronómico aporta el 4\% al PIB nacional cerca de 32 billones, además NARANJO-ARANGO R., NARANJO-AFRICANO G., LEONES-VIANA A. R. 
LA GASTRONOMÍA COLOMBIANA. ATRACTIVO TURÍSTICO EN CRECIMIENTO

en la gerenación de empleo aportó un 6\% que según (Dinero, 2015) se habla de un estimativo de 400,000 puestos de trabajo, sin mencionar a los proveedores y los productores agrícolas que cada vez más estan ganando protagonismo en este sector, para el año 2017 se estima que la proyección del sector comercio, donde se encuentra la gastronomía será del 2\%, no hay duda que cada vez más éste sector está tomando fuerza y consolidándose como uno de los que mas proyecciones tendrán en años venideros.

Analizando la información anterior, es posible afirmar que gracias al turismo, la gastronomía ha ido ganando un protagonismo cada vez más creciente dentro de la economía del país, y es precisamente gracias a los turistas que llegan a conocer y degustar la gastronomía nacional, se mueve mucho más el sector culinario en las distintas regiones.

La actividad turística tiene un fuerte impacto sobre la economía de Colombia, debido a que es un país rico en biodiversidad, cultura, y poseer tantos patrimonios históricos, lo han convertido en un sector que está jalonando favorablemente los indicadores económicos, fortaleciendo de una forma u otra todo el engranaje de los sectores económicos del país, de acuerdo con (El tiempo, 2017) en el año 2016 Illegaron al país 5’092,000 turistas, lo que representó un aumento del 12,6\% respecto al año anterior, para el año 2017, el sector turismo se convirtió en el segundo generador de divisas en Colombia, superando los 6’500,000 visitantes, según (El tiempo, 2018).

Un alto porcentaje de éstos turistas, llegó a conocer a Colombia por su gastronomía, pues en su mayoría según (Colombia.co, 2017) fueron los platos, las técnicas de elaboración, la historia de cada plato, los puntos mas atrayentes de turistas al país, y el sector sigue con proyecciones muy positivas frente a tal situación, pues según (Dinero, 2016) las ventas de alimentos y bebidas en el país van a crecer de forma sostenida en los próximos cinco años hasta 35.000 millones de dólares en 2020. Considerando lo expuesto anteriormente, es evidente que el turismo guarda una estrecha relación con la gastronomía, porque dentro de los diferentes atractivos que oferta la actividad turística en el país, se puede encontrar el turismo de eventos, festividades y el gastronómico que cada vez más está siendo explotado y potencializado.

\section{Turismo gastrónomico en Colombia. Un análisis de sus regiones}

El país cuenta con seis regiones: Amazonía, Andina, Caribe, Insular, Orinoquía y Pacífico, cada una de ellas cuenta con una cultura, costumbres, y sobre todo con cocina totalmente distinta, aunque en algunos casos la base sea la misma, la preparación de cada plato, ingredientes, NARANJO-ARANGO R., NARANJO-AFRICANO G., LEONES-VIANA A. R. 
LA GASTRONOMÍA COLOMBIANA. ATRACTIVO TURÍSTICO EN CRECIMIENTO

hacen del arte culinario diferente en cada región del país y es que son precisamente las tradiciones y costumbres diferentes que hacen de la gastronomía colombiana un ejemplar a nivel mundial, porque en cada plato se aprecia un poco de la cultura que es representativa de la región, de la localidad, y esto es clave para el turista porque a él le gusta compenetrarse con el lugar que visita, para hacer su estancia lo mas amena posible, trata de estar inmerso en todo lo que se hace, de tal forma que se siente parte del lugar, y esto marca ese momento en su memoria, llevándose así una imagen positiva y agradable de la región que visita.

Colombia ofrece una amplia variedad de opciones gastronómicas en todas sus regiones, la experiencia de conocer todo el proceso que abarca la elaboración de los platos en cada rincón del país, es un hecho que resulta bastante importante e interesante, según (Díaz \& Pabón, 2015).

La experiencia de poder conocer el proceso de preparación, permite la interacción y conocimiento de la calidad de la gastronomía, y sin duda éste aspecto motiva a los turistas que buscan cada vez mas experiencias diferentes para salir de la rutina, del mismo modo este proceso sirve para ayudar a entender y conocer la riqueza cultural que posee la nación, así lo aseveran (Naranjo \& Leones, 2017); como lo expresa la literatura, que la experiencia del turismo gastronómico está asociado al disfrute, en particular que atrae a los turismas a viajar atraídos por disfutar las bondades de la gastronomía de cada lugar que visitan.

El hecho de ser Colombia un país multicultural, ha permitido que por sus reconocidas fiestas, festivales, lleguen y visiten muchas personas de otros lugares, platos como la bandeja paisa, la lechona tolimense, el sancocho costeño, el ajiaco, el arroz con coco, son algunos de los platos mas comunes en la comida colombiana, y al exterior se han podidio llevar tanto así, que ya han sido creados algunos restaurantes en otros países llevando así una imagen de Colombia a otras partes. Sin embargo, esto constituye un riesgo, porque cuando en el exterior se piensa en un restaurante Colombiano no es fácil asociarlo a un plato o menú típico, sino aparecen una gama de platos y preparaciones como representación del país.

De acuerdo con un artículo publicado en (El tiempo, 2016) Colombia posee una gran variedad de alimentos y preparaciones en su gastronomía, esto gracias a la diversidad de productos que se dan en las regiones, a esto se le suma las tradiciones propias de cada lugar y los 48 eventos gastronómicos que se llevan a cabo en todo el país, lo que se convierte en un potencial para el desarrollo turístico y gastronómico del país. 


\section{LA GASTRONOMÍA COLOMBIANA. ATRACTIVO TURÍSTICO EN CRECIMIENTO}

A continuación, se muestra una tabla que contiene los platos más representativos de la comida colombiana, diferenciado claramente en cada una de las regiones.

\section{Tabla 1}

Principales platos de la gastronomía Colombiana

\begin{tabular}{|c|c|c|}
\hline Región & Nombre del plato & Ciudad \\
\hline Amazonía & $\begin{array}{l}\text { Pataresca } \\
\text { Mojojoy } \\
\text { Casabe }\end{array}$ & Leticia \\
\hline Andina & $\begin{array}{c}\text { Ajiaco } \\
\text { Lechona } \\
\text { Hormigas culonas } \\
\text { Bandeja paisa } \\
\text { Carantanta } \\
\text { Aplanchado } \\
\text { Arepa paisa } \\
\text { Sopa de mute }\end{array}$ & $\begin{array}{l}\text { Bogotá } \\
\text { Ibagué } \\
\text { Bucaramanga } \\
\text { Medellín } \\
\text { Cauca } \\
\text { Popayán } \\
\text { Medellín } \\
\text { Bucaramanga }\end{array}$ \\
\hline Caribe & $\begin{array}{l}\text { Arroz con coco } \\
\text { Sancochos } \\
\text { Arroz con mariscos } \\
\text { Mondongo } \\
\text { Pasteles } \\
\text { Area de huevo } \\
\text { Bollo de yuca } \\
\text { Butifarra }\end{array}$ & $\begin{array}{c}\text { Cartagena } \\
\text { Toda la región } \\
\text { Cartagena } \\
\text { Sincelejo } \\
\text { Sincelejo } \\
\text { Luruaco } \\
\text { Barranquilla } \\
\text { Barranquilla }\end{array}$ \\
\hline Insular & $\begin{array}{c}\text { Rondón } \\
\text { Bacalao con papas } \\
\text { Arroz con mariscos } \\
\text { Pescado en Escapeche }\end{array}$ & San Andrés Isla \\
\hline Orinoquía & $\begin{array}{c}\text { Majuele } \\
\text { Periquillo } \\
\text { Picillo } \\
\text { Tungo } \\
\text { Pez amanónico }\end{array}$ & $\begin{array}{l}\text { Villavicencio } \\
\text { Villavicencio } \\
\text { Arauca } \\
\text { Yopal } \\
\text { Villavicencio }\end{array}$ \\
\hline Pacífica & $\begin{array}{c}\text { Caldo de denton salpreso } \\
\text { Crema de cabeza de langostinos } \\
\text { Caldo de gazapo el arroz } \\
\text { Atollao de carne ahumada } \\
\text { Arroz atollado } \\
\text { Pandebono } \\
\text { Cuy }\end{array}$ & $\begin{array}{l}\text { Chocó } \\
\text { Chocó } \\
\text { Chocó } \\
\text { Chocó } \\
\text { Cali } \\
\text { Cali } \\
\text { Pasto }\end{array}$ \\
\hline
\end{tabular}

Fuente: Recuperado de Naranjo y Leones (2017).

En los últimos años, la gastronomía del país ha tenido una expansión a nivel internacional, y como lo afirman (Naranjo \& Leones, 2017) dicha expansión ha llevado a que los productos 
LA GASTRONOMÍA COLOMBIANA. ATRACTIVO TURÍSTICO EN CRECIMIENTO

elaborados por las manos colombianas vayan logrando cierto grado de participación en los mercados extranjeros, toda vez que cuentan con originalidad, creatividad y sobre todo la calidad. Asimismo, se habla que los platos colombianos son portadores de un sabor único, y no es para menos, pues el país goza de amplias zonas aptas para el cultivo de los diferentes insumos que son utilizados para la elaboración de los platos, que finalmente son una excelente combinación entre trabajo campesino y manos expertas en cocina tradicional.

\section{Potencialiades del turismo gastronómico colombiano}

Lo anterior, permite afirmar que la cocina de Colombia está despegando como destino culinario, puesto que los sabores, enamoran y cautivan a todos, siempre dejan buenos recuerdos, por ello se dice que son experiencias culinarias únicas (Colombia.co, 2014). La gastronomía es una forma de hacer turismo en el país, de acuerdo con (Colombia.co, 2014) la gastronomía colombiana cautiva a mas de 4 millones de turistas extranjeros que visitan el país anualmente, entonces considerando ésta cifra tan importante es necesario seguir desarrollando estrategias que puedan ser útiles para continuar pontencializando este sector que tanto aporta al país.

En el año 2013, según datos del Ministerio de Comercio, Industria y Turismo, cerca de 17 millones de personas viajaron alrededor del país, cifra que aumentó en un 10\% sobre el año 2011 (Pachón, 2014), estas movilizaciones de turistas en el país, que aunque no tienen fines directamente gatronómicos, si pueden generar motivaciones secundarias en los visitantes hacia el consumo de alimento, conllevando indirectamente a un turismo gastronómico. Dentro de los destinos más concurridos, se encontraron Bogotá, Cartagena y Medellín, en primer lugar Bogotá porque se ha convertido en un epicentro de negocios, el $57 \%$ de los turistas nacionales y extranjeros escogieron esta ciudad como destino turistico, Cartagena por su arquitectura, historia y patrimonio, y Medellín por la red de museos que atraen a los turistas, del mismo modo se puede mencionar la ciudad de Cali, que ofrece como platos fuerte la gastronomía y la música, tan solo en el 2013 visitaron la ciudad 980,000 personas, y sobre todo la oferta gastronómica ha permitido un aumento del $20 \%$ con relación a la llegada de los turistas (Pachón, 2014) dichas cifras se han convertido en un aliciente que continua motivando al país para obtener un mayor progreso. 
LA GASTRONOMÍA COLOMBIANA. ATRACTIVO TURÍSTICO EN CRECIMIENTO

\section{CONCLUSIONES}

Es claro el potencial económico que tiene el sector gastronómico para Colombia; por lo que se convertiría en una decisión estratégica de país convertir este sector con potencial de posicionamiento y exportación. Es necesario apostar a la gastronomía, para convertirla en una de las principales ventajas competitivas, debido a que el país tiene todos los elementos y herramientas necesarias para hacerlo, alimentos de primera calidad, técnicas de preparación, el personal con la experiencia, la originalidad e innovación en cada plato, solo resta empoderarse mas de ésta situación para conseguir resultados a gran escala (Portafolio, 2014). Aunado a lo anterior, la misma diversidad geográfica y cultural se refleja en la riqueza que poseen los platos tradicionales. La comida de las regiones, permite conocer un poco acerca de la cultura, las tradiciones propias del lugar; éste aspecto cada vez mas está llamando la atención del turista, puesto que se ha notado últimamente un aumento significativo en lo que respecta al turismo gastronómico; es decir, todos aquellos turistas que viajan a conocer otros lugares, atraídos principalmente por el arte culinario representativo de la región. Considerando la diversidad de platos y multiculturalidad gastronómica del país, es importante diseñar estrategias de identificación y reconocimiento, a fin de generar platos que se conviertan en representación gastronómica del país hacia el exterior, con el propósito de generar una identifidad y marca en este tema. El turismo es uno de los principales pilares del sector de gastronomía en el país, en la actualidad, funcionan perfectamente bajo una estrecha relación, habiendo cifras que respaldan bien a aquellos que vienen a conocer Colombia; lo hacen en gran medida por la diversidad culinaria y sobretodo por la excelencia e idiosincrasia de cada plato. Cada vez más está llegando turistas, el país se observa como un destino seguro, cómodo, y sobre todo atractivo para todo aquel que quiera vivir una experiencia diferente. Para finalizar, teniendo en cuenta todas las herramientas, técnicas que se tienen en materia de arte gastronómico, es hora de explotarlas al máximo, el país ya está siendo reconocido a nivel internaiconal, y es por ello, que se debe aprovechar todo ese auge, a fin de hacer de Colombia un país líder en turismo gastronómico.

\section{REFERENCIAS BIBLIOGRÁFICAS}

Bogotá turismo. (2016). Turismo gastronómico. Recuperado el 15 de Julio de 2017, de http://www.bogotaturismo.gov.co/Turismo\%20Gastron\%C3\%B3mico

Colombia.co. (1 de Septiembre de 2014). La gastronomía, una oportunidad para el turismo en Colombia. Recuperado el 15 de Julio de 2017, de http://www.colombia.co/esta-escolombia/cultura/la-gastronomia-una-oportunidad-para-el-turismo-en-colombia/ 
LA GASTRONOMÍA COLOMBIANA. ATRACTIVO TURÍSTICO EN CRECIMIENTO

Colombia.co. (25 de Julio de 2017). La gastronomía, una oportunidad para el turismo en Colombia. Recuperado el 9 de Abril de 2018, de http://www.colombia.co/esta-escolombia/cultura/la-gastronomia-una-oportunidad-para-el-turismo-en-colombia/

Díaz, J., \& Pabón, A. (Septiembre de 2015). Turismo gastronómico. Caribeña de Ciencias Sociales. Recuperado el 15 de Julio de 2017, de http://www.eumed.net/rev/caribe/2015/09/gastronomia.html

Dinero. (6 de Junio de 2013). La gastronomía como estrategia de turismo. Recuperado el 12 de Julio de 2017, de http://www.dinero.com/pais/articulo/la-gastronomia-como-estrategiaturismo/177310

Dinero. (20 de Marzo de 2015). El negocio gastronómico está en su punto. Recuperado el 14 de Julio de 2017, de http://www.dinero.com/edicion-impresa/negocios/articulo/inversionesproyectos-gastronomicos-colombia/206929

Dinero. (27 de Agosto de 2016). Gastronomía colombiana está en crecimiento. Recuperado el 9 de Abril de 2018, de http://www.elcampesino.co/gastronomia-colombiana-estacrecimiento/

El espectador . (2018 de Febrero de 2018). Colombia rompe récord con 6,5 millones de turistas extranjeros en 2017. El espectador. Recuperado el 9 de Abril de 2018, de https://www.elespectador.com/economia/colombia-rompe-record-con-65-millones-deturistas-extranjeros-en-2017-articulo-738272

El tiempo. (5 de Junio de 2010). Lo que buscan los turistas extranjeros que llegan a Bogotá. Recuperado el 15 de Julio de 2017, de http://www.eltiempo.com/archivo/documento/CMS-7740550

El tiempo. (11 de Marzo de 2016). Los servicios financieros fueron los que más crecieron en el 2015. Recuperado el 15 de Julio de 2017, de http://www.eltiempo.com/archivo/documento/CMS-16533751

El tiempo. (26 de Noviembre de 2016). Turismo y gastronomía, la puesta de Colombia. Recuperado el 15 de Julio de 2017, de http://www.eltiempo.com/vida/viajar/gastronomiatipica-de-colombia-40338

El tiempo. (27 de Septiembre de 2017). El turismo, un sector en crecimiento. El tiempo. Recuperado el 24 de Marzo de 2018, de http://www.eltiempo.com/vida/viajar/crecimiento-del-turismo-en-colombia-135304

El tiempo. (30 de Enero de 2018). Las positivas cifras que muestran el ascenso del turismo en Colombia. El tiempo. Recuperado el 24 de Marzo de 2018, de http://www.eltiempo.com/vida/viajar/turismo-en-colombia-crecio-en-el-2017-176846

Feo Parrondo, F. (2005). Turismo gastronómico en Asturias. Cuadernos de turismo, 15, 77 - 96. Recuperado el 30 de Julio de 2017, de http://revistas.um.es/turismo/article/view/18501

Gaona, C. (1999). La alimentación como variable a tener en cuenta en los estudios de desarrollo rural . En Alimentación y Cultura (pp. 749-764). Huesca: La Val de Onsera.

Garzón, D. (13 de Junio de 2015). El sector gastronómico creció 22\% en el último año con 90.000 restaurantes. Recuperado el 14 de Julio de 2017, de 
LA GASTRONOMÍA COLOMBIANA. ATRACTIVO TURÍSTICO EN CRECIMIENTO

https://www.larepublica.co/ocio/el-sector-gastronomico-crecio-22-en-el-ultimo-ano-con90000-restaurantes-2266206

Hall, M., \& Mitchell, R. (2002). Tourism as a force for gastronomic globalization and localization. En A. M. Hjalager, \& G. Richards, Tourism and Gastronomy (págs. 71 - 90). London: Routledge.

Hall, M., Sharples, L., Mitchell, R., Macionis, N., \& Cambourne, B. (2003). Food tourism around the world. Development, management and markets. Oxford: Butterworth-Heinemann.

López-Guzmán , T., \& Sánchez Cañizares, S. (2012). La gastronomía como motivación para viajar. Un estudio sobre el turismo culinario en Córdoba. Pasos. Revista de Turismo y patrimonio Cultural, 10(5), 575 - 584. Recuperado el 31 de Julio de 2017, de www.redalyc.org/articulo.oa?id=88124507012

Mincomercio. (5 de Septiembre de 2012). Gastronomía colombiana, impulsora de turismo y empleos. Recuperado el 14 de Julio de 2017, de http://www.mincit.gov.co/publicaciones/4212/gastronomia_colombiana_impulsora_de_tur ismo_y_empleos

Mitchell, R., \& Hall, C. (2003). Consuming tourists: food tourism consumer behaviour. En M. C. Hall, L. Sharples, R. Mitchell, \& N. y. Macionis, Food Tourism Around the World. Development, Management and Markets (págs. 60 - 80). Oxford: ButterworthHeinemann.

Naranjo, R., \& Leones, A. (22 de Junio de 2017). Variedad del turismo gastronómico en Colombia. México.

Pachón, V. (23 de Octubre de 2014). Bogotá, Cartagena y Medellín, los destinos preferidos. La república. Recuperado el 19 de Julio de 2017, de https://www.larepublica.co/economia/bogota-cartagena-y-medellin-los-destinospreferidos-2184181

Portafolio. (23 de Septiembre de 2014). Por la estrategia de una "cocina colombiana". Recuperado el 15 de Julio de 2017, de http://www.portafolio.co/tendencias/estrategiacocina-colombiana-45608

Quan, S., \& Wang, N. (2004). Towards a structural model of the tourist experience:an illustration. Tourism Management, 25, 297 - 305. Recuperado el 30 de Julio de 2017, de http://www.sciencedirect.com/science/article/pii/S0261517703001304

Ruíz, L. M. (24 de Enero de 2013). El negocio de la gastronomía viene aumentando su buen sabor. La república. Recuperado el 19 de Julio de 2017, de https://www.larepublica.co/ocio/el-negocio-de-la-gastronomia-viene-aumentando-subuen-sabor-2030051

Vanguardia.com. (7 de Octubre de 2016). Restaurantes del país aportan 4\% al PIB. Recuperado el 14 de Julio de 2017, de http://www.vanguardia.com/economia/nacional/375667-restaurantes-del-pais-aportan-4al-pib 